\title{
STRAINED INDIUM ARSENIDE/GALLIUM ARSENIDE LAYER FOR QUANTUM CASCADE LASER DESIGN USING GENETIC ALGORITHM
}

\author{
David Mueller \\ Dr. Gregory Triplett, Dissertation Supervisor
}

\begin{abstract}
Achieving high power, continuous wave, room temperature operation of midinfrared (3-5 um) lasers is diffcult due to the effects of auger recombination in band-to-band designs. Intersubband laser designs such as quantum cascade lasers reduce the effects of recombination, increasing efficiency and have advantages in large tunability of wavelength ranges. Highly efficient quantum cascade laser designs are typically used in lasers designed for $>5 \mathrm{um}$ wavelength operation due to the small offset of conduction band energy in lattice matched materials. Some promising material systems have been used to achieve high-power output in the first atmospheric window (3-5 um) but still suffer from low efficiency. Larger 1conduction band offset is attainable through the use of strained materials. However, these material systems have limitations on the traditional (100) crystal orientation due to the large strain and low critical thickness. The necessity for controlled two-dimensional, optical quality layer growth limits the amount of strain incorporation due to defect formation in highly lattice mis-matched layers. The material systems used in this study are GaAs (100) and (111)B, AlGaAs, and (Ga)InAs. In the initial stage of research, I found that pseudomorphic growth of highly strained InAs layers on GaAs (111)B is possible. However, the growth window is very narrow and necessitates precise control over growth temperature and anion overpressure to achieve optical quality layers. As a result, a second stage of research explores the design space made available by this finding by using genetic algorithm based design and simulation of devices with a Schrodinger-Poisson solver.
\end{abstract}

